\title{
Föderalismus und Rechtsstaat - ein Vergleich der Länder am Bodensee
}

\section{Arnold Marti *}

Föderalismus und Rechtsstaatlichkeit gelten heute als wichtige und bewährte Staats- und Verfassungsprinzipien. Ein Vergleich der Länder am Bodensee zeigt, dass Herkunft, Entwicklung und heutige Ausgestaltung dieser Prinzipien auch auf engem Raum recht unterschiedlich sind, aber stets eine gegenseitige Beeinflussung stattgefunden hat und sich heute in den betroffenen vier Staaten nebst Unterschieden auch ganz ähnliche Fragen und Herausforderungen stellen.

I. Einleitung. 115

II. Föderalismus 115

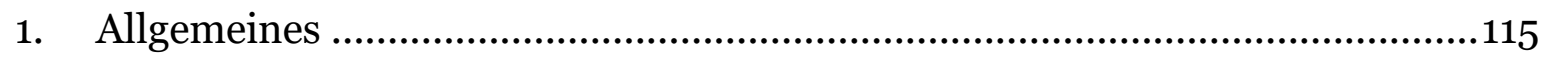

2. Tradition des Föderalismus .................................................................116

3. Merkmale und Unterschiede im Föderalismus ...................................... 118

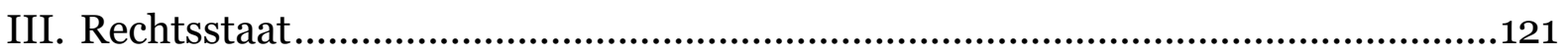

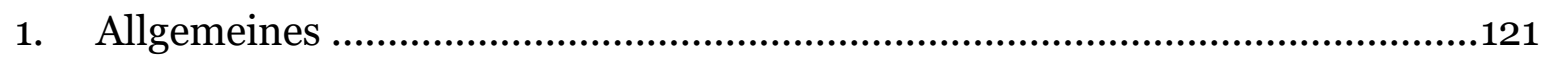

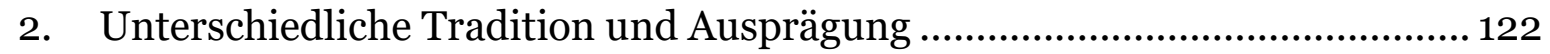

3. Bedeutung der Europäischen Menschenrechtskonvention.......................... 125

IV. Besondere Fragen und Herausforderungen im Verhältnis von

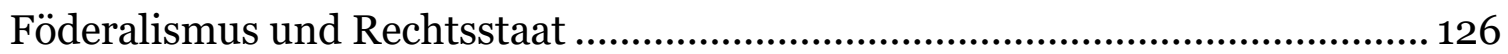

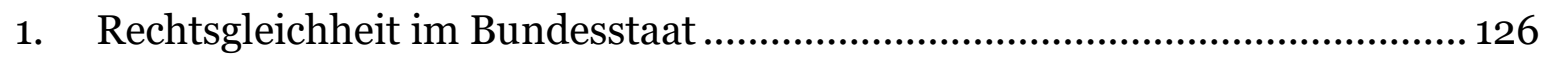

2. Rechtsstaatliche Defizite in kleinräumigen Verhältnissen .......................... 128

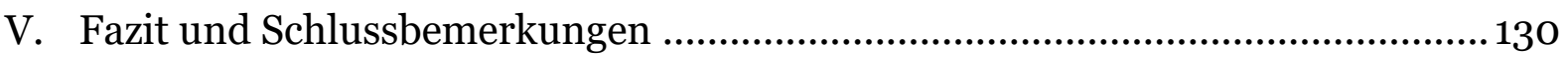

Zitiervorschlag: Arnold Marti, Föderalismus und Rechtsstaat - ein Vergleich der Länder am Bodensee, in: sui-generis 2020, S. 114

URL: $\quad$ sui-generis.ch/125

DOI: $\quad$ https://doi.org/10.21257/sg.125

* Titularprofessor für Staats- und Verwaltungsrecht an der Universität Zürich; ehemaliger Vizepräsident des Schaffhauser Obergerichts (marti@anwalt-sh.ch). Beim vorliegenden Text handelt es sich um die schriftliche, mit Anmerkungen ergänzte Fassung des am 21. September 2019 am 67. Treffen der Juristinnen und Juristen der Bodenseeländer in Schaffhausen gehaltenen Vortrags. Der Stil des mündlichen Referats wurde weitgehend beibehalten. 


\section{Einleitung}

1 An den seit Anfang der 1950er-Jahren stattfindenden, von den örtlichen Anwalts- und Juristenverbänden organisierten Jahrestreffen, welche Juristinnen und Juristen aller Berufsrichtungen der Vierländerregion Bodensee ${ }^{1}$ vereinigen, wird unter uns Fachkolleginnen und Fachkollegen immer wieder diskutiert, welche Unterschiede bzw. Vorteile und Nachteile in unseren unterschiedlichen staatsrechtlichen Systemen rund um den Bodensee bestehen und wie sich diese auf unser Berufsleben, aber auch auf das allgemeine Zusammenleben auswirken. In den letzten Monaten hatte ich mich überdies für ein Sammelwerk zum 20. Jahrestag des Inkrafttretens der neuen Schweizer Bundesverfassung vom 18. April 1999 am 1. Januar 2020 intensiv mit dem Thema des Verhältnisses von «Föderalismus» und «Rechtsstaat» zu beschäftigen. ${ }^{2}$ So lag es für mich nahe, für die ehrenvolle Aufgabe, den heutigen Festvortrag zu halten, ein Thema in diesem Bereich zu wählen und den Versuch zu wagen, einen Vergleich zwischen den Ländern am Bodensee anzustellen. Ich beschränke mich hierbei grundsätzlich auf die beiden erwähnten Staatsprinzipien, doch besteht auch ein enger Bezug zum bedeutsamen dritten Staatsprinzip,

\footnotetext{
Direkt an den Bodensee grenzen nur Deutschland, Österreich und die Schweiz. Das Fürstentum Liechtenstein ist jedoch über den Alpenrhein mit dem Bodensee nahe verbunden und gehört touristisch und politisch zur Bodenseeregion; es ist auch Mitglied der 1972 gegründeten Internationalen Bodensee-Konferenz (IBK).

2 Vgl. dazu das nun erschienene dreibändige Werk «Verfassungsrecht der Schweiz», hrsg. von Oliver Diggelmann, Maya Hertig Randall und Benjamin Schindler, Zürich/Basel/Genf 2020. Mein dortiger Beitrag trägt den Titel «Verhältnis von Föderalismus und Rechtsstaatlichkeit»; siehe Band I, S. $595 \mathrm{ff}$.
}

der Demokratie.3 Diesbezüglich haben wir am Bodensee ja interessantes Anschauungsmaterial: direkte oder halbdirekte Demokratie in der Schweiz (mit den Volks- und Ständereferenden in der Gesetzgebung), indirekte bzw. parlamentarische Demokratie in Deutschland und Österreich, konstitutionelle Erbmonarchie auf demokratisch-parlamentarischer Grundlage im Fürstentum Liechtenstein. Bemerkenswert ist, dass damit auf engem Raum praktisch alle in der neueren Geschichte vorkommenden und allgemein anerkannten Staatsformen vertreten sind. 4

\section{Föderalismus}

\section{Allgemeines}

2 Doch nun zuerst zum Föderalismus: Abgeleitet vom lateinischen «foedus» (der Bund) wird darunter das staatliche Organisationsprinzip verstanden, bei dem den einzelnen Gliedstaaten (Kantone in der Schweiz, Bundesländer in Deutschland und Österreich) eine begrenzte Eigenständigkeit und Staatlichkeit mit einer entsprechender Behördenorganisation (Regierung, Parlament, Gerichte) und eigenen Gesetzen zukommt, wobei die Gliedstaaten zusammen den Gesamt-

$3 \overline{\text { Vgl. zu Staatsprinzipen Demokratie, Föderalis- }}$ mus und Rechtsstaatlichkeit Ulrich Häfelin/ Walter Haller/Helen Keller/Daniela Thurnherr, Schweizerisches Bundesstaatsrecht, 9. Aufl., Zürich/Basel/Genf 2016, Rz. 168 ff., und zahlreiche Beiträge in Giovanni Biaggini/Thomas Gächter/ Regina Kiener (Hrsg), Staatsrecht, 2. Aufl., Zürich/St. Gallen 2015, sowie in Band I und II des in Fn. 2 erwähnten Sammelwerkes.

4 Vgl. zu den heute vorkommenden Staatsformen Walter Haller/Alfred Kölz/Thomas Gächter, Allgemeines Staatsrecht, 5. Aufl., Basel 2013, Rz. 115 ff. und Andreas Glaser in Biaggini/Gächter/ Kiener (Fn. 3), § 3 Rz. 1 ff. 
staat (einen sog. Bundesstaat) bilden.5 Dies gilt für die ausdrücklich so bezeichnete Bundesrepublik Deutschland, aber auch für die Republik Österreich, welche dies - evtl. aus historischen Gründen? unterschlägt und auch für die Schweiz. Dies obwohl deren formelle Bezeichnung «Schweizerische Eidgenossenschaft» wohl ebenfalls aus historischen Gründen - eher auf einen blossen Staatenbund hinweist, wo es keinen einheitlichen Gesamtstaat mit entsprechenden Behörden gibt und welcher dementsprechend nicht Gesetze erlässt, sondern grundsätzlich nur vertragliche Rechtsakte kennt. Bei den drei grösseren Staaten um den Bodensee handelt es sich nach dem Gesagten allesamt um sog. föderale bzw. Bundesstaaten.

Das Gegenteil dazu bildet ein Einheitsoder Zentralstaat, welcher über eine einheitliche Regierung, Verwaltung und Gesetzgebung für das ganze Land verfügt. ${ }^{6}$ Auch hierfür findet sich am Bodensee ein Beispiel: das Fürstentum Liechtenstein, welches dieses Jahr sein 300-jähriges Bestehen feiern kann (Zusammenfügung und Anerkennung als Reichsfürstentum durch Kaiser Karl VI im Jahr 1719). Es besteht zwar ebenfalls aus zwei Landschaften (Ober- und Unterland) und insgesamt 11 Gemeinden. Diesen kommt zwar aufgrund von Art. 110 der Liechtensteiner Verfassung vom 5. Oktober 1921 ausdrücklich verwaltungsmässige Autonomie zu, doch handelt es sich nicht um

$5 \overline{\text { Vgl. zum Begriff und Wesen des Föderalismus }}$ bzw. des Bundesstaats und zum Unterschied zu einem blossen Staatenbund Haller/Kölz/Gächter (Fn. 4), Rz. 500 ff. und Andreas Kley in Biaggini/ Gächter/Kiener (Fn. 3), § 10 Rz. 4 ff.

6 Vgl. dazu Haller/Kölz/Gächter (Fn. 4), Rz. 489 ff. und Andreas Kley in Biaggini/Gächter/Kiener (Fn. 3), § 10 Rz. 11 ff. eigentliche Gliedstaaten, sondern nur um Organe der verwaltungsmässigen Dezentralisation des im Übrigen einheitlichen Staates. Etwas anderes wäre ja auch nicht sinnvoll, handelt es sich doch mit rund 38‘00o Einwohnern (etwa gleich viel wie die Stadt Schaffhausen) um den kleinsten deutschsprachigen Staat (aber immerhin nur den sechstkleinsten Staat weltweit). ${ }^{7}$

\section{Tradition des Föderalismus}

4 Die drei ganz unterschiedlich grossen Bundesstaaten Schweiz, Deutschland und Österreich sind demgegenüber traditionell sehr föderalistisch: Deutschland war seit dem Mittelalter bis 1806, als das Heilige Römische Reich Deutscher Nation aufgelöst wurde, ein Staatenbund und damit ein Flickenteppich von über 300 miteinander durch Verträge verbundene Klein- und Mittelstaaten, welchen erst im 19. Jahrhundert sukzessive und im Rahmen mehrere kriegerischer Auseinandersetzungen die Nationsbildung in Form des Deutschen Reiches gelang. ${ }^{8}$ Auch das Deutsche Reich (1871-1918) umfasste jedoch 25 zum Teil weiter unterteilte Teilstaaten (22 Monarchien und drei Republiken sowie das Reichsland ElsassLothringen). Diese Teilstaaten blieben in der Weimarer Republik (1918-1933) nun in demokratischer Form und in verringerter Zahl (18) - bestehen, und mit weniger Rechten als im Kaiserreich und in der heutigen Bundesrepublik. Auch in der Nazi-Diktaturzeit blieben die Länder formell bestehen, wurden aber zu aus-

7 Vgl. dazu auch Themen-Dossier «300 Jahre Liechtenstein» in der NZZ vom 21. September 2019.

8 Vgl. zur Geschichte des deutschen Föderalismus und der Einigung Deutschlands Albert Funk, Föderalismus in Deutschland. Vom Fürstenbund zur Bundesrepublik, Paderborn 2010. 
führenden Organen der Reichsbehörden degradiert. In der Bundesrepublik bestehen heute (nach der Wiedervereinigung im Jahr 1990) 16 Bundesländer, 11 alte Bundesländer im Westen (inkl. Berlin) und 5 neue Bundesländer im Osten. Das kleinste Bundesland ist Bremen (680'00o Einwohner), das grösste NordrheinWestfalen mit rund 18 Mio. Einwohner. Aber auch die beiden BodenseeanrainerBundesländer sind je bevölkerungsreicher und im Fall von Bayern auch flächenmässig grösser als die ganze Schweiz: Bayern mit rund 13 Mio. Einwohnern und das 1952 aufgrund eines komplizierten Entscheidungsprozesses zusammengefügte Bundesland BadenWürttemberg mit rund 11 Mio. Einwohnern (mit Eingliederung auch der früher preussischen Hohenzollern-Gebiete Hechingen und Sigmaringen).

5 Auch in Österreich hat der Föderalismus Tradition, war das alte Österreich doch ein Vielvölkerstaat, der vom Bodensee an die Adria und entlang der Donau bis fast ans Schwarze Meer reichte. ${ }^{9}$ Die 1804 neu gegründete Erbmonarchie war zwar formell-rechtlich ein Einheitsstaat, in dem lediglich die 17 traditionellen österreichischen Kronländer gewisse Selbstbestimmungsrechte hatten (daher wurde teilweise auch von «Völkerkerker» gesprochen). Erst 1867 erfolgte mit dem österreichisch-ungarischen Ausgleich ein Umbau zu einem eigentlichen Staatenverband (Cisleithanien [Österreich mit den alten Kronländern] und Transleithanien [Ungarn mit dessen südlichen $\mathrm{Ne}$ benländern]; beide Teile der Doppelmo-

\footnotetext{
Vgl. zur Entwicklung von der Donaumonarchie zur heutigen Republik Österreich Klaus Berchtold, Verfassungsgeschichte der Republik Österreich, Wien 1998 und Wilhelm Brauneder, Österreichische Verfassungsgeschichte, 11. Aufl., Wien 2009.
}

narchie benannt nach dem Grenzfluss Leitha zwischen Wien und Budapest). Als nach dem Zerfall der Habsburgermonarchie am Ende des Ersten Weltkrieges und der Unterzeichnung des Vertrages von St. Germain am 19. September 1919 die neue kleine Alpenrepublik Österreich gegründet wurden, bekamen die in diesem Gebiet bestehenden früheren Kronländer den Status von Bundesländern, wobei neben dem Burgenland und dem nun selbständigen Wien auch unser Nachbarland Vorarlberg dazu kam, welches lange zu Tirol gehörte und 1919 aufgrund einer Volksabstimmung der Schweiz hätte beitreten wollen (was diese jedoch ablehnte). ${ }^{10}$ Kleinstes Bundesland ist heute das Burgenland mit knapp 300'00o Einwohnern, grösstes Wien mit 1,9 Mio. Einwohnern.

6 Ein urföderalistisches Land ist natürlich auch die Schweiz: ${ }^{11}$ Diese entstand nach der Mythologie aus dem Rütlischwur von 1291 in den drei Urkantonen am Vierwaldstättersee (als Schutzbündnis gegen Habsburger Feudalherren, die ja ursprünglich aus der heutigen Schweiz kamen [Aargau]), und wuchs bis zum Ende des Mittelalters zur 13-örtigen Alten Eidgenossenschaft an, welche erst 1648 mit dem westfälischen Frieden formell aus dem Heiligen Römischen Reich Deutscher Nation austrat. Das Feudalsystem der alten Eidgenossenschaft brach 1798

10 Vgl. zur Wiederaufnahme dieser Frage in St. Gallen nach 100 Jahren den Artikel «Vorarlberg als Schweizer Kanton?», in St. Galler Tagblatt vom 3. November 2019.

11 Zum Schweizer Föderalismus siehe Häfelin/ Haller/Keller/Thurnherr (Fn. 3), Rz. 934 ff.; zur Entstehung und Ausgestaltung des schweizerischen Föderalismus Kley (Fn. 6), § 10 Rz. 1 ff., $\S 11$ Rz. 1 ff. und Rainer J. Schweizer, Entstehung und Entwicklung des schweizerischen Föderalismus, im erwähnten Sammelwerk (Fn. 2), Band I, S. $531 \mathrm{ff}$. 
(ähnlich wie wenige Jahre später das Reich [siehe den Reichsdeputationshauptbeschluss von 1803]) unter dem Ansturm der französischen Revolutionstruppen zusammen. Die Franzosen bildeten mit schweizerischen Verbündeten für fünf Jahre den Einheitsstaat «Helvetische Republik» nach französischem Modell. Dies funktionierte aber nicht. Schon 1803 erarbeitete Napoleon in Paris mit Schweizer Delegierten die sog. Mediationsverfassung, welche die alten Kantone im Sinne eines Staatenbundes wiederherstellte und überdies den bisherigen Untertanengebieten (darunter auch Thurgau und St. Gallen) Kantonsstatus verlieh, so wie später 1815 der Wienerkongress den französischsprachigen Gebieten Wallis, Neuenburg und Genf. Der französischsprachige Berner Jura erstritt sich erst in der zweiten Hälfte des letzten Jahrhunderts als jüngster Kanton diesen Status (Annahme in der eidg. Volksabstimmung vom September 1978). ${ }^{12} \mathrm{Zu}$ einem eigentlichen Bundesstaat wurde die Schweiz erst 1848, als die Kantone nach dem Sieg der Bundesstaatsanhänger im Sonderbundskrieg von November 1847 (letzte militärische Auseinandersetzung auf Schweizer Boden) der neuen Bundesverfassung - nur mit SiegerMehrheit und mit zum Teil fragwürdigen Methoden (Nichtstimmende wurden z.B. in Luzern den Ja-Stimmen zugerechnet) - zustimmten $\left(15^{1 / 2}\right.$ gegen $6^{1 / 2}$ Stände). ${ }^{13}$ Heute besteht die Schweiz aus 26 Kantonen (20 Vollkantone und sechs Halbkantone [hervorgegangen aus der

12 Zur Schaffung des Kantons Jura vgl. Häfelin/ Haller/Keller/Thurnherr (Fn. 3), Rz. 997 ff.

13 Vgl. dazu Häfelin/Haller/Keller/Thurnherr (Fn. 3), Rz. 48 ff. und Andreas Kley, Geschichte des schweizerischen Verfassungsrechts von 1798 bis in die Gegenwart, in dem in Fn. 2 erwähnten Sammelwerk, Band I, S. 57 ff., Rz. 31 ff. politisch oder religiös begründeten Aufteilung von Vollkantonen]). Kleinster Kanton ist Appenzell Innerrhoden mit heute rund 16'00o Einwohnern, grösster der Kanton Zürich mit immerhin 1,52 Mio. Einwohnern.

\section{Merkmale und Unterschiede im Föderalismus}

7 Neben der ganz unterschiedlichen Geschichte und Grösse der betroffenen Territorien weisen Deutschland, Österreich und die Schweiz alle wichtigen Merkmale von Bundesstaaten auf. Typisch ist zunächst das aus den USA stammende bundesstaatliche Zweikammersystem, wonach das Parlament zwei unterschiedlich zusammengesetzte Kammern aufweist: eine Volksvertretung zusammengesetzt entsprechend dem Bevölkerungsanteil der einzelnen Gliedstaaten und eine Ländervertretung, wo jeder Gliedstaat im Normalfall gleich stark vertreten ist. ${ }^{14}$ In Deutschland der Bundestag und der Bundesrat, in Österreich der Nationalrat und der Bundesrat und in der Schweiz der National- und der Ständerat. Idealiter werden beide Kammern vom Volk gewählt und es kommen ihnen gleiche Kompetenzen zu (vollkommenes Zweikammersystem), wie dies heute grundsätzlich für die Schweiz zutrifft. In Deutschland besteht der Bundesrat dagegen aus weisungsgebundenen Vertretern der Landesregierungen, und es kommen ihm abgestufte Kompetenzen zu (etwa sog. Zustimmungs- bzw. blosse Einspruchsgesetze). In Österreich werden die Mitglieder des Bundesrates von den Länderparlamenten gewählt und ha-

14 Vgl. dazu und zu den verschiedenen Modellen des Zweikammersystems Haller/Kölz/Gächter (Fn. 4), Rz. 837 ff. und Häfelin/Haller/Keller/Thurnherr (Fn. 3), Rz. $1438 \mathrm{ff}$. 
ben in der Gesetzgebung - von wenigen Ausnahmen abgesehen (z.B. Länderkompetenzeinschränkungen in Verfassungsgesetzen) - nur ein suspensives Veto, das der Nationalrat durch einen neuen Beschluss überwinden kann. In beiden Ländern ist die Stimmkraft der Ländervertretungen überdies als Besonderheit nach der Grösse der Bundesländer abgestuft. ${ }^{15}$

Neben dem Zweikammersystem ist entsprechend dem Grundgedanken der Subsidiarität - die Kompetenzvermutung zugunsten der Länder in der Gesetzgebung Wesensmerkmal von Bundesstaaten. ${ }^{16}$ Gesetzgebungskompetenzen des Gesamtstaates bedürfen daher immer einer Grundlage in der Verfassung. Diese verfassungsmässige Kompetenzvermutung zugunsten der Gliedstaaten besteht denn auch in allen drei Bundesstaaten um den Bodensee (siehe Art. 3 der Bundesverfassung der Schweizerischen Eidgenossenschaft vom 18. April 1999 [BV], Art. 70 des Grundgesetzes für die Bundesrepublik Deutschland vom 23. Mai 1949 [GG] und Art. 15 Abs. 1 des Bundesverfassungsgesetzes der Republik Österreich vom 1. Oktober 1920 [B-VG], wobei allerdings in Deutschland und in Österreich faktisch bzw. aufgrund entsprechender Verfassungsregelungen sehr viele Gesetzgebungszuständigkeiten beim Bund liegen. Aber auch in der Schweiz hat in den letzten Jahrzehnten aufgrund der Mobilitätsbedürfnisse von Wirtschaft und Gesellschaft eine zunehmende Rechtsvereinheitlichung oder zumindest eine Rechtsharmonisierung stattgefunden,

$15 \overline{\text { Vgl. dazu Haller/Kölz/Gächter (Fn. 4), Rz. 840 ff., }}$ $847 \mathrm{ff}$.

16 Vgl. dazu auch Haller/Kölz/Gächter (Fn. 4), Rz. 559 ff. und Häfelin/Haller/Keller/Thurnherr (Fn. 3), Rz. 1049 ff. teilweise auch auf dem Konkordatsweg (Verträge zwischen den Kantonen; Bsp.: nur teilweise geglückte Harmonisierung der Baubegriffe und des Volksschulwesens). Die Austarierung von Gesetzgebungszuständigkeiten und Verantwortlichkeiten bildet denn auch in allen drei Bundesstaaten immer wieder Gegenstand von Föderalismusreformen: In der Schweiz wurde 2004 durch eine umfangreiche Verfassungs-Teilrevision der Finanzausgleich und die Aufgabenverteilung zwischen Bund und Kantonen im Sinne klarer Grundsätze neu geregelt (NFA: Neugestaltung des Finanzausgleichs und der Aufgabenteilung). Ähnlich sind in Deutschland die Gesetzgebungskompetenzen von Bund und Kantonen durch die Föderalismusrevision von 2006 neu geregelt worden, wobei aber eine Reform der Finanzverfassung ausgeklammert wurde. Auch in Österreich besteht hinsichtlich der Verteilung von Kompetenzen und Finanzen zwischen Bund und Ländern Reformbedarf, doch sind bisherige Reformversuche (z.B. der ÖsterreichKonvent von 2003-2005) gescheitert. ${ }^{17}$

9 Ebenfalls charakteristisch für Bundesstaaten - auch im Sinne einer bundesstaatlichen Existenzsicherung - ist, dass gesamtstaatliche Verfassungsänderungen regelmässig nur durch Zustimmung von Volks- und Länderkammer möglich sind, in Deutschland und in Österreich (dort aber nur sofern die Länderkompetenzen

$17 \mathrm{Zu}$ aktuellen Föderalismusreformen in der Schweiz, Deutschland und Österreich vgl. Haller/ Kölz/Gächter (Fn. 4), Rz. 587 ff., für Deutschland Hans-Peter Schneider, Der neue deutsche Bundesstaat. Bericht über die Umsetzung der Föderalismusreform I, Baden-Baden 2013, für Österreich die Webseite des Österreich-Konvents und für die Schweiz Marti (Fn. 2), Rz. 4 sowie Häfelin/ Haller/Keller/Thurnherr (Fn. 3), Rz. 74a, 970 ff., 1051. 
betroffen sind) grundsätzlich sogar nur mit einer Zweidrittelsmehrheit in beiden Kammern (in Österreich allenfalls vorbehältlich einer Volksabstimmung bei Gesamtrevision oder auf Verlangen von einem Drittel einer Parlamentskammer; in Deutschland überdies teilweise Ewigkeitsgarantie für einzelne Bestimmungen des Grundgesetzes). In der Schweiz ist entsprechend der direktdemokratischen Staatsform für Verfassungsänderungen immer eine Mehrheit von Volk und Ständen in der Volksabstimmung erforderlich. ${ }^{18}$

Neben diesen grundsätzlichen Übereinstimmungen gibt es aber auch wesentliche Unterschiede im föderalen System der drei Bundesstaaten am Bodensee, welche auch mit den erwähnten Besonderheiten zusammenhängen. So soll der Bundesstaat etwa in Österreich - möglicherweise als Folge der monarchischeinheits-staatlichen Geschichte im Habsburgerreich des 19. Jahrhunderts - möglichst einheitlich in Erscheinung treten, weshalb die Gesetzgebungskompetenzen schwergewichtig dem Bund zugewiesen werden (sog. unitarischer Föderalismus). Den Bundesländern kommen vor allem Gesetzgebungskompetenzen in Materien mit Lokalbezug (Bau- und Planungsrecht, Tourismus) zu, während z.B. die Gerichtsbarkeit (abgesehen von den 2014 geschaffenen Länderverwaltungsgerichten) ganz Bundessache ist und - anders als in der Schweiz und in Deutschland auch vom Bund, nicht den Ländern getragen wird. ${ }^{19}$

$18 \overline{\text { Vgl. dazu Haller/Kölz/Gächter (Fn. 4), Rz. } 367 \mathrm{ff}}$. und für die Schweiz insbesondere Häfelin/Haller/ Keller/Thurnherr (Fn. 3), Rz. 1754 ff.

19 Vgl. dazu Marti (Fn. 2), Rz. 3. Abgesehen von der Gerichtsbarkeit wird aber auch in Österreich anders als in den USA - das Bundesrecht von den
11 Um die Unterschiede zwischen den Gliedstaaten eher klein zu halten, können Machtbeziehungen zwischen Bund und Gliedstaaten auch eng miteinander verknüpft werden, wie dies im sog. kooperativen Föderalismus der Fall ist. ${ }^{20}$ Dieser spielt in Form der Konkordate (Verträge zwischen den Kantonen; Ausbau mit NFA-Revision) und der 1993 gegründeten Konferenz der Kantonsregierungen (Interessenvertretung gegenüber dem Bund), aber auch der schon länger bestehenden Fachdirektorenkonferenzen auch in der Schweiz eine zunehmende Rolle. In Deutschland geht dies aber darüber hinaus, indem auch die Verfassungsorgane selber für diese Zusammenarbeit besonders organisiert werden. So besteht die Länderkammer in Deutschland - wie erwähnt - nicht aus frei gewählten Mitgliedern, sondern aus Vertretern der Landesregierungen, welche bei der Gesetzgebung z.T. ein Zustimmungs-, z.T. ein Einspruchsrecht haben. Besondere Mitwirkungsrechte des Bundesrates bestehen überdies teilweise auch beim Verordnungsrecht und bei verwaltungsinternen Bundesvorschriften sowie bei der Rechtsetzung auf EU-Ebene.

12 Im Fall der Schweiz wird dagegen von einem symmetrischen Föderalismus gesprochen, weil Bund und Kantone im Prinzip über die gleichen Rechte, je zahlreiche Kompetenzen und eine je voll ausgebaute Staatsorganisation verfügen, womit sich aber auch umfassend und für

Bundesländern, nicht von dezentralen Organen der Bundesverwaltung vollzogen (sog. europäischer Vollzugsföderalismus); vgl. dazu Haller/ Kölz/Gächter (Fn. 4), Rz. 549 ff.

${ }^{20}$ Zum kooperativen Föderalismus allgemein und insbesondere für Deutschland und die Schweiz Marti (Fn. 2), Rz. 3 und Haller/Kölz/Gächter (Fn. 4), Rz. 594 f. 
beide Ebenen die Frage des Verhältnisses von Föderalismus und Rechtsstaat stellt. ${ }^{21}$ Weil in der Schweiz die Kantone überdies - anders als die Bundesländer in Deutschland und Österreich - ein eigenes, inzwischen allerdings auch - aber nur formell (begrifflich und verfahrensrechtlich) - durch Bundesrecht harmonisiertes Einkommens- und Vermögenssteuerrecht verfügen, ist auch von Wettbewerbs- oder Fiskalföderalismus die Rede. Tatsächlich besteht von Kanton zu Kanton für Bürgerinnen und Bürger sowie juristische Personen eine sehr unterschiedliche Steuerbelastung, die allenfalls ohne Weiteres mehr als das Doppelte betragen kann (was natürlich enorme Auswirkungen auf Bodenpreise, Bevölkerungsstruktur und Infrastrukturstandard hat). In Deutschland dagegen besteht für die Gemeinden und Länder nur bei der Gewerbesteuer ein gewisser Spielraum für den Steuersatz. Die in der Schweiz aktuell auf internationalem Druck hin durchgeführte und nach einem ersten gescheiterten Anlauf nun am 19. Mai 2019 vom Volk abgenommene Steuerreform (Abschaffung der ermässigten Besteuerung von Holding- und Domizilgesellschaften) könnte den Steuerwettbewerb in der Schweiz noch verschärfen, weshalb auch der Ruf nach einer Eingrenzung dieses Wettbewerbs durch eine materielle Steuerharmonisierung ertönt, was bis-

21 Vgl. dazu Marti (Fn. 2), Rz. 3 und eingehend Denise Brühl-Moser, Schweizerischer Föderalismus im internationalen Vergleich, in dem in Fn. 2 erwähnten Sammelwerk, Band I, S. 565 ff.; zur trotz NFA feststellbaren Konzeptionsarmut des schweizerischen Föderalismus auch Eva Maria Belser, Föderalismuskonzeption der Bundesverfassung, in dem in Fn. 2 erwähnten Sammelwerk, Band I, S. 667 ff., insbesondere Rz. 8 f. her aber von Parlament und Volk stets abgelehnt wurde. ${ }^{22}$

\section{Rechtsstaat}

\section{Allgemeines}

13 Zum Rechtsstaat: Während der Föderalismus der territorialen Machtbegrenzung dient, soll mit der Rechtsstaatlichkeit, zu der sich heute alle vier Länder also auch Liechtenstein - in ihren Verfassungen zum Teil ausdrücklich, zum Teil sinngemäss bekennen, die staatliche Gewalt durch das Recht gebunden und damit die Freiheit der Einzelnen, allenfalls auch die Autonomie von Gebietskörperschaften, gesichert werden. Ein einheitlicher Rechtsstaatsbegriff besteht aber - wie auch beim Föderalismus nicht. Es wird vielmehr von einem «partiell offenen Strukturprinzip» gesprochen. ${ }^{23}$ Übereinstimmung besteht immerhin darin, dass formelle und materielle Gehalte des Rechtsstaatsprinzips zu unterscheiden sind. Gesetzmässigkeit, Gewaltenteilung und richterlicher Rechtsschutz gelten als formelle Seite des Rechtsstaats, weil sie Zuständigkeit, Organisation und Verfahren betreffen. Die Grundrechte (allenfalls auch Sozialrechte oder zumindest Sozialziele), die Rechtsgleichheit, das Verhältnismässigkeitsprinzip und das Willkürverbot bilden die

$22 \overline{\text { Vgl. zum ausgeprägten Finanzföderalismus in der }}$ Schweiz Brühl-Moser (Fn. 21), Rz. 53; zur im Mai 2019 angenommenen Steuerreform die Homepage des Eidg. Finanzdepartements zum Thema «Steuerreform und AHV-Finanzierung (STAF)»; zur anlässlich der Volksabstimmung vom 28. November 2010 abgelehnten Volksinitiative «Für faire Steuern. Stopp dem Missbrauch beim Steuerwettbewerb (Steuergerechtigkeits-Initiative)» die Botschaft des Bundesrates vom 6. März 2009 (BBl 2009, S. 1907 ff.).

23 Vgl. dazu auch Marti (Fn. 2), Rz. 5 mit zahlreichen Hinweisen. 
materielle, inhaltliche Seite der Rechtsstaatlichkeit. Namentlich aus diesen inhaltlichen Anforderungen ergibt sich auch, dass das in Deutschland geprägte Rechtsstaatskonzept deutlich über das angelsächsische «Rule of law» (Herrschaft des Rechts)-Prinzip hinausgeht. ${ }^{24}$

\section{Unterschiedliche Tradition und Ausprägung}

14 Während beim Föderalismus in den Bodenseeländern - wie erläutert - eine alte Tradition besteht, kann dies beim Rechtsstaat nicht gesagt werden. Hier geht es in allen besprochenen Ländern eher um eine Reaktion auf eine autokratische Tradition in teils seit dem Mittelalter bestehenden monarchischen und aristokratischen, teils auch zünftischen Verhältnissen (wie hier in Schaffhausen), wobei diese Reaktion allerdings - wie der Föderalismus - in den einzelnen Staaten zu unterschiedlichen Zeiten und in unterschiedlicher Form erfolgte bzw. wirksam wurde.

Im Heiligen Römischen Reich Deutscher Nation gab es schon im Spätmittelalter Ansätze der Rechtsstaatlichkeit. Hinzuweisen ist vor allem auf das 1495 geschaffene Reichskammergericht, welches primär für die Einhaltung des Landfriedens aber auch eine gewisse Rechtseinheit und einen minimalen Rechtsschutz für alle Untertanen zu sorgen hatte. ${ }^{25}$ Formalisiert und als solche begründet wurde die Rechtsstaatsidee aber im 19. Jahrhundert in Deutschland. Der Tübinger Rechtsund Staatswissenschaftsprofessor Robert

$24 \overline{\text { Vgl. zur Geschichte, zu den Elementen und unter- }}$ schiedlichen Konzepten des Rechtsstaats auch Haller/Kölz/Gächter (Fn. 4), Rz. 444 ff.

25 Vgl. dazu die Hinweise bei Astrid Epiney in Basler BV-Kommentar (2015), Art. 5 Rz. 17. von Mohl (1799-1875), welcher 1848 Mitglied der Frankfurter Nationalversammlung war, prägte diesen Begriff als Antithese zum autoritären Polizeistaat. Nach dem Scheitern der 1848-Revolution konzentrierte sich das liberale Bürgertum in Deutschland darauf, die monarchisch dominierte Regierung und Verwaltung rechtlich zu binden. Dies spielte im 19. Jahrhundert beim Aufbau eines eigentlichen Verwaltungsrechts mit geordnetem Verfahrensrecht eine wichtige Rolle ${ }^{26}$. In der Weimarer Verfassung und im Bonner Grundgesetz wurde das Rechtsstaatsprinzip weiterentwickelt. Schon die Weimarer Verfassung von 1919 verankerte die Rechtsgleichheit, enthielt einen Grundrechtskatalog und sah die Gewaltenteilung vor. Allerdings bestand kein Schutz gegen Verfassungsänderungen durch Parlament und Regierung, was der Weimarer Verfassung nach der NSMachtergreifung die Wirkung entzog. ${ }^{27}$

16 Bei der Schaffung des Bonner Grundgesetzes im Jahre 1949 bildete diese Erfahrung stetes Mahnmal. Das Rechtsstaatsprinzip wurde daher in verschiedener Hinsicht perfektioniert. ${ }^{28}$ Besonders zu erwähnen ist die umfassende Rechtsweggarantie von Art. 19 Abs. 4 GG (Grundrecht auf wirksamen Rechtsschutz gegen alle Akte der öffentlichen Gewalt), welche

$26 \overline{\text { Vgl. dazu Epiney (Fn. 25), Art. } 5 \text { Rz. 17, Benjamin }}$ Schindler in: St. Galler BV-Kommentar (3. Aufl. 2014), Art. 5 Rz. 3 ff. und ders., Entstehung und Entwicklung der Rechtsstaatsidee in der Schweiz, in dem in Fn. 2 erwähnten Sammelwerk, Band II, S. 907 ff., Rz. 2 mit weiteren Hinweisen.

27 Vgl. dazu und zum Zusammenbruch des deutschen Rechtsstaats in der Nazizeit die eindrücklichen Berichte von Horst Dreier und Walter Pauly in: Veröffentlichungen der Vereinigung der Deutschen Staatsrechtslehrer, Band 60, Berlin/ New York 2001, S. 9 ff., 73 ff.

28 Vgl. dazu auch Haller/Kölz/Gächter (Fn. 4), Rz. 466 ff. 
in dieser Form auch im internationalen Vergleich völlig neu war. Das Rechtsstaatsprinzips selber wurde sodann in Art. 20 Abs. 3 GG in neuartiger und umfassender Weise umschrieben (eigentlicher rechtsstaatlicher Schlüsselsatz): «Die Gesetzgebung ist an die verfassungsmässige Ordnung, die vollziehende Gewalt und die Rechtsprechung sind an Gesetz und Recht gebunden», woraus sich auch ergibt, dass Rechtsstaatlichkeit nicht einfach mit dem bestehenden, positiven Gesetzesrecht gleichgesetzt werden kann. Besondere Beachtung verdient auch, dass gewisse rechtsstaatliche Grundanforderungen aufgrund der Erfahrungen der Weimarer-Zeit bzw. der anschliessenden NS-Diktatur unabänderlich erklärt wurden (Ewigkeits-Garantie für Staatsform und wichtige Verfassungsprinzipien; vgl. Art. 79 Abs. 3 GG). So etwas ist weder im Schweizer noch im österreichischen Verfassungsrecht vorgesehen. In Österreich wird ganz auf die erforderliche parlamentarische Zweidrittelsmehrheit und in der Schweiz auf die erforderliche Zustimmung von Volk und Ständen vertraut. ${ }^{29}$ Eine eigentliche Bewährungsprobe für die Ewigkeitsgarantie im deutschen Grundgesetz hat sich bisher zum Glück noch nie ergeben. Für die Abgrenzung und den Schutz der Garantie im Einzelnen ist im Übrigen das Bundesverfassungsgericht in Karlsruhe zuständig, welches insoweit auch über dem Gesetzgeber steht. ${ }^{\circ}$

29 Vgl. dazu auch Haller/Kölz/Gächter (Fn. 4), Rz. 367.

30 Zur besonderen Stellung und Bedeutung des deutschen Verfassungsgerichts vgl. Haller/Kölz/ Gächter (Fn. 4), Rz. 990 ff., Stephan Detjen, Das Bundesverfassungsgericht zwischen Recht und Politik, Aus Politik und Zeitgeschichte, Heft 3738/2001, S. 3 ff., sowie verschiedene Beiträge zum 60-Jahr-Jubiläum dieser Institution in: Aus
17 Abgefärbt hat die deutsche Rechtsstaatsdiskussion im 19. Jahrhundert auch auf die Entwicklung in Österreich. Hinzuweisen ist vor allem auf die sog. Dezemberverfassung von 1867 in Österreich, welche im Zusammenhang mit dem bereits erwähnten österreichisch-ungarischen Ausgleichs geschaffen wurde (sie galt freilich nur für die cisleithanischen, d.h. nichtungarischen Länder). ${ }^{31}$ Dieses «Staatsgrundgesetz über die allgemeinen Rechte der Staatsbürger» sah ausdrücklich die Rechtsgleichheit und die wichtigsten auch heute noch auf dieser Grundlage geltenden Freiheitsrechte vor (u.a. persönliche Freiheit, Versammlungsfreiheit, Pressefreiheit, Glaubensund Gewissensfreiheit, Wissenschaftsund Lehrfreiheit; vgl. den Verweis in Art. 149 des heutigen B-VG, dessen erste Fassung 1920 erlassen wurde). Gleichzeitig wurde das bis 1918 bestehende Reichsgericht und ein Gesetz über die Unabhängigkeit der Justiz (mit Einrichtung eines Verwaltungsgerichtshofes) und über die Ausübung der Regierungs- und Vollzugsgewalt geschaffen. Im heutigen Art. 18 Abs. 1 B-VG ist nun auch das Gesetzmässigkeitsprinzip für die gesamte staatliche Verwaltung vorgeschrieben. ${ }^{2}$

18 In der Schweiz war die Rechtsstaatlichkeit in den früheren Bundesverfassungen von 1848 und 1874 nur mässig ausge-

Politik und Zeitgeschichte, Heft 35-36/2011, S. $3 \mathrm{ff}$.

31 Vgl. dazu Helmut Paul Wallner, Dezemberverfassung 1867. Entstehung, Inhalt und Auswirkung, München 2008.

32 Vgl. dazu Marcus Hrncir/Sigrid Urbanek, Der demokratische Rechtsstaat Österreich. Allgemeine Darstellung der österreichischen Rechtsordnung und Grundprinzipien der Bundesverfassung, in Forum Politische Bildung (Hrsg.), Justiz - Recht - Staat. Sonderband der Informationen zur Politischen Bildung, Innsbruck/Wien 1999, S. $7 \mathrm{ff}$. 
prägt; der Begriff kam im Verfassungswortlaut auch nicht vor.33 Im Vordergrund stand die Zusammenfügung zu einer bundesstaatlichen Ordnung und die Begrenzung der Behördenmacht wurde vor allem auf dem Weg der demokratischen Mitwirkung gesehen (Volkswahl der Regierungen und zum Teil auch der Gerichte in den Kantonen; Gesetzesreferendum in Bund und Kantonen). Eine personelle Gewaltentrennung im Verhältnis Bundesparlament/Bundesgericht bestand erst ab 1874 und im Bundesverwaltungsrecht war der Bundesrat (die Bundesregierung) lange Zeit wichtigste Rechtsprechungsbehörde. Gegen kantonale Gesetzgebungs- und Verwaltungsakte bestand dagegen ab 1875 die sog. staatsrechtliche Beschwerde ans Bundesgericht, womit vor allem kantonale Willkür ausgeschlossen und die staatsrechtlichen Anforderungen an die Kantone durchgesetzt werden sollten. Ein unabhängiger kantonaler Verwaltungsrechtsschutz wurde in der überwiegenden Zahl der Kantone erst nach dem Zweiten Weltkrieg geschaffen. Auch gegenüber der Bundesverwaltung wurde ein unabhängiger Rechtsschutz erst ab 1969 eingeführt (mit vielen Ausnahmen). 34

33 Vgl. zur wechselvollen Geschichte des Rechtsstaatsbegriffs in Politik und Staatsrecht der Schweiz sowie zu dessen später Durchsetzung erst ab den 1980er-Jahren Benjamin Schindler, Entstehung und Entwicklung der Rechtsstaatsidee in der Schweiz, in dem in Fn. 2 erwähnten Sammelwerk, Band II, S. $907 \mathrm{ff}$.

34 Zur späten Einführung einer umfassenden unabhängigen Verwaltungsgerichtsbarkeit in der Schweiz und der Auffangfunktion der früheren staatsrechtlichen Beschwerde ans Bundesgericht vgl. Arnold Marti, Die aktuelle Justizreform Abschluss einer über hundertjährigen Entwicklung hin zur umfassenden Verwaltungsgerichtsbarkeit in der Schweiz, in: Roger Zäch u.a. (Hrsg.), Individuum und Verband. Festgabe zum Schweizerischen Juristentag 2006, Zürich/Basel/ Genf 2006, S. 505 ff.
19 Heute (seit der Justizreform in der Verfassung [auch diese vom Volk im Jahr 2000 angenommen]) gilt für Bund und Kantone eine Rechtsweggarantie (Art. 29a BV), welche aufgrund der Gesetzgebung grundsätzlich nur für «vorwiegend politische Akte» eingeschränkt werden kann. Umgesetzt wurde die Rechtsweggarantie auf Bundesebene durch das Bundesgerichtsgesetz vom 17. Juni 2005 (BGG) und die Schaffung von neuen erstinstanzlichen Bundesgerichten: Bundesverwaltungsgericht und Bundespatentgericht in St. Gallen, Bundesstrafgericht in Bellinzona.35 Zurzeit befindet sich das BGG wieder in Revision; umstritten ist der Ausnahmekatalog für die Beschwerden ans Bundesgericht und die vom Bundesgericht gewünschte Abschaffung der subsidiären Verfassungsbeschwerde, welche heute auch dann eine Anrufung des Bundesgerichts gegen kantonale Entscheide ermöglicht, wenn kein ordentliches Rechtsmittel ans Bundesgericht zur Verfügung steht (wichtig auch als Damoklesschwert für die kantonale Justiz, vor allem in bestehenden kleinräumigen Verhältnissen). 36

$35 \overline{\text { Vgl. zur im Jahr } 2000 \text { von Volk und Ständen }}$ angenommenen Justizreform mit Einführung einer grundsätzlich uneingeschränkten Rechtsweggarantie Marti (Fn. 2), Rz. 8 f., Häfelin/Haller/ Keller/Thurnherr (Fn. 3), Rz. 70 ff. und Alain Griffel, Rechtsschutz, in dem in Fn. 2 erwähnten Sammelwerk, Band II, S. 1073 ff., insbesondere Rz. 16 ff., $24 \mathrm{ff}$.

$36 \mathrm{Vgl}$. zu diesem inzwischen gescheiterten Gesetzgebungsprojekt die Botschaft des Bundesrates vom 15 Juni 2018 (BBl 2018, S. 4605 ff.) und dazu kritisch Rainer J. Schweizer, Reform der Bundesgerichtsbarkeit, in «Justice - Justiz - Giustizia» 2018/4. Nachdem der Nationalrat die Vorlage mit verschiedenen Änderungen verabschiedet hat (u.a. Beibehaltung der subsidiären Verfassungsbeschwerde), ist der Ständerat am 17. Dezember 2019 nicht auf die Vorlage eingetreten, welchem Beschluss der Nationalrat am 5. März 2020 gefolgt ist. 
Der Rechtsschutz wurde im Übrigen aufgrund der Justizreform im Zivil- und Strafrecht (nicht aber im Verwaltungsrecht) gesamtschweizerisch vereinheitlicht (Schweizerische Zivil- und Strafprozessordnung, in Kraft seit 1. Januar 2011).37 Auch ein Grundrechtskatalog bestand in den früheren Bundesverfassungen nicht oder nur in Ansätzen. Vorgesehen war die Rechtsgleichheit und das Willkürverbot sowie die Wirtschaftsfreiheit und die Glaubens- und Gewissenfreiheit; im Übrigen wurden die Grundrechte lange Zeit weitgehend den Kantonen überlassen. Das Bundesgericht entwickelte im 20. Jahrhundert aber zahlreiche Freiheitsrechte (politische Freiheiten; Eigentumsgarantie) aus der geltenden Rechts- und Demokratieordnung. Die neue Bundesverfassung von 1999 bedeutet auch in dieser Hinsicht einen Quantensprung. Es gibt jetzt einen Grundrechtskatalog und minimale Sozialziele; ferner sind verschiedene rechtsstaatliche Grundsätze nun ausdrücklich in der Verfassung festgehalten (so das Gesetzmässigkeitsprinzip, Anforderungen an Grundrechtsbeschränkungen, Grundsatz von Treu und Glauben, Beachtung des Völkerrechts). ${ }^{8}$

37 Vgl. dazu die Hinweise bei Marti (Fn. 2), Rz. 9 Im Vergleich der Bodenseeländer sehr negativ fällt das hohe Kostenrisiko vor allem im vereinheitlichten Zivilprozess auf, wobei dies in einer anlaufenden ersten Gesetzesrevision (hoffentlich) korrigiert werden kann; vgl. dazu Griffel (Fn. 35), Rz. 36 ff. mit weiteren Hinweisen.

38 Zur Entwicklung des Grundrechtsschutzes in der Schweiz und zu den heute in der Bundesverfassung bestehenden Grundrechten vgl. Marti (Fn. 2), Rz. 6 f., Häfelin/Haller/Keller/Thurnherr (Fn. 3), Rz. 205 ff. und insbesondere die Beiträge von Jörg Paul Müller und Regina Kiener in dem in Fn. 2 erwähnten Sammelwerk, S. 1167 ff. und $1193 \mathrm{ff}$.

\section{Bedeutung der Europäischen Menschenrechtskonvention}

21 Erwähnt werden muss, dass die Fortentwicklung des Rechtsstaats in den europäischen Ländern und insbesondere in der Schweiz eng zusammenhängt mit der Schaffung bzw. dem Beitritt zur Europäischen Menschenrechtskonvention (mit wichtigen Grundrechts- und Rechtsschutzgarantien). Die Schweiz ist bekanntlich dieser Konvention erst 1974 beigetreten, nachdem sie im Rahmen von Volksabstimmungen endlich das Frauenstimmrecht eingeführt und die aus der Kulturkampfzeit stammenden religiösen Ausnahmeartikel abgeschafft hatte. Beim Rechtsschutz bestand ursprünglich ein Vorbehalt zugunsten der in der Schweiz lange Zeit üblichen verwaltungsinternen Rechtspflege, doch stellte sich heraus, dass dieser Vorbehalt die Anforderungen der Rechtsprechung des Europäischen Gerichtshofes für Menschenrechte (EGMR) nicht erfüllte, was zur Beschleunigung der Einführung eines flächendeckenden unabhängigen Verwaltungsrechtsschutzes in der Schweiz führte (Entscheide EGMR Belilos und Weber 1988 bzw. 1990).39 Aber auch im Zivilund namentlich im Strafprozessrecht brachte die EMRK für die Schweiz wichtige rechtsstaatliche Neuerungen. $\mathrm{Zu}$ erwähnen ist etwa, dass im Kanton Schaffhausen das Obergerichts ein von der alten Strafprozessordnung abweichendes, provisorisches Haftrecht direkt gestützt auf die EMRK erliess, welches von Ende 1977 bis zum Inkrafttreten der neuen

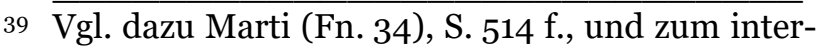
nationalen Grundrechtsschutz (inkl. die UNOPakte I und II); eingehend Jörg Künzli und Maya Hertig Randall im in Fn. 2 erwähnten Sammelwerk, Band II, S. 1245 ff. und 1267 ff. 
kantonalen Strafprozessordnung im Herbst 1988 Anwendung fand. 40

Während der Verwaltungsrechtsschutz in der Schweiz heute wie auch in Deutschland und Österreich voll ausgebaut ist, besteht in der Schweiz weiterhin ein rechtsstaatliches Defizit hinsichtlich des Rechtsschutzes gegenüber dem Bundesgesetzgeber. Dessen Akte können - anders als Gesetzgebungsakte in Deutschland und Österreich - gemäss dem Anwendungsgebot von Art. 190 BV durch das Bundesgericht als Höchstgericht weder direkt noch im Anwendungsfall (d.h. akzessorisch) ausser Kraft gesetzt werden. ${ }^{41}$ Auch eine beschränkte akzessorische Prüfung wurde vom Parlament im Rahmen der Justizreform nach 2000 abgelehnt. Daher ist es besonders wichtig, dass Volk und Stände im November 2018 die sog. Selbstbestimmungsinitiative abgelehnt haben, mit welcher u.a. die Geltung der EMRK in der Schweiz hätte eingeschränkt werden sollen. Die Ablehnung dieser Initiative ermöglicht es, dass das Bundesgericht zumindest EMRK-widrigen Bundesgesetzen weiterhin die Anwendung versagen kann; weiterhin möglich bleibt in solchen Fällen auch die Anrufung des EGMR.42

40 Vgl. dazu Arnold Marti, Die Bedeutung der EMRK in der Rechtsprechung der kantonalen Gerichte - am Beispiel des Kantons Schaffhausen, in: Samantha Besson/Eva Maria Belser (Hrsg.), Die Europäische Menschenrechtskonvention und die Kantone, BENEFRI-Tagung im Europarecht des Instituts für Europarecht, Zürich/Basel/Genf 2014, S. 95 ff., insbesondere S. 99 ff.

41 Zur fehlenden Verfassungsgerichtsbarkeit gegenüber dem schweizerischen Bundesgesetzgeber vgl. Griffel (Fn. 35), Rz. 30 ff. und dazu sowie zur Situation in den Nachbarländern auch Haller/ Kölz/Gächter (Fn. 4), Rz. 968 ff., insbesondere Rz. 1013.

42 Vgl. dazu Häfelin/Haller/Keller/Thurnherr (Fn. 3), Rz. 2086 ff., Griffel (Fn. 35), Rz. 31 und zur am

\section{Besondere Fragen und Herausfor- derungen im Verhältnis von Föderalismus und Rechtsstaat}

23 Im Zusammenspiel von Föderalismus und Rechtsstaat stellen sich verschiedene interessante, aber auch heikle Rechtsfragen und Herausforderungen. Ich erwähne etwa die Durchsetzung der föderalistischen und rechtsstaatlichen Grundprinzipien im Bundesstaat (wofür in der Schweiz das besondere Gewährleistungsverfahren gemäss Art. $51 \mathrm{Abs} .2 \mathrm{BV}$ durch das Bundesparlament besteht), das Erfordernis bundesstaatlicher Kollisionsregeln für die Rechtsanwendung (mein seinerzeitiges Habilitationsthema), Fragen der Grundrechtskonkurrenz zwischen Bund und Gliedstaaten (z.B. die aktuell umstrittene Volksinitiative «Grundrechte für Primaten» in Basel-Stadt). Aus Zeitgründen kann ich auf diese interessanten Fragen hier nicht näher eingehen.43 Kurz eintreten will ich aber noch auf zwei interessante Fragen im Verhältnis Rechtsstaat/Föderalismus, welche auch für die juristische Alltagspraxis bedeutsam sind: die Rechtsgleichheit im Bundesstaat und mögliche rechtsstaatliche Defizite in kleinräumigen Strukturen.

\section{Rechtsgleichheit im Bundesstaat}

24 Ein Grundproblem im Verhältnis Rechtsstaat/Föderalismus stellt sich bei der

25. November 2018 von Volk und Ständen abgelehnten Volksinitiative «Schweizer Recht statt fremde Richter (Selbstbestimmungsinitiative)» die Botschaft des Bundesrates vom 5. Juli 2017 (BBl 2017, S. 5355 ff.); zum Verhältnis Völkerrecht/Landesrecht in Deutschland und andern Länder auch den Bericht des Bundesrates vom 5. März 2010 (BBl 2010, S. 2263 ff., insbesondere S. 2290 ff.) und für Liechtenstein Patricia M. Schiess Rütimann, Die Stellung der EMRK in Liechtenstein, Jusletter vom 4. Februar 2019.

43 Vgl. dazu nun die eingehenden Ausführungen bei Marti (Fn. 2), Rz. 16 ff. 
Rechtsgleichheit, dieser zentralen rechtsstaatlichen Anforderung. Wenn den Gliedstaaten eigene Gesetzgebungskompetenzen eingeräumt werden, wie dies zum Föderalismus gehört, muss auch in Kauf genommen werden, dass in den entsprechenden Bereichen keine Gleichheit besteht. Insoweit kann der föderalistische Staatsaufbau dann auch als «offene Flanke der Gleichheit» bezeichnet werden. Die Frage ist dann, wie weit die Regelungen in den Gliedstaaten auseinander gehen dürfen.44 In Deutschland besteht nach Grundgesetz für den Bereich der konkurrierenden Gesetzgebung und auch für die Verteilung der Finanzmittel der Auftrag, für die Gleichwertigkeit der Lebensverhältnisse im Bundesgebiet zu sorgen, was mit der Wiedervereinigung 1990 noch verstärkt wurde (Art. 72 Abs. 2 und Art. 106 Abs. 3 GG), aber - wie wir heute nach 30 Jahren wissen - eine sehr offene Formulierung darstellt und nur beschränkt gelungen ist. In der Schweiz mit ihrem - wie bereits erwähnt - sehr kompetitiven Föderalismus gab es lange Zeit keine ähnliche Regelung. Mit der NFA-Föderalismusreform 2004 wurde in der neuen Aufgabenverteilungsnorm aber der ebenfalls etwas unbestimmte Grundsatz aufgenommen, dass Leistungen der Grundversorgung allen Personen in vergleichbarer Weise offen stehen müssen (Art. 43a BV). Dieser Grundsatz wurde bisher namentlich für die Wasser- und Energieversorgung, die Kommunikationsinfrastruktur und den öffentlichen Verkehr angerufen; er trägt gerade in letzterem Bereich viel zur Attraktivität der Schweiz bei und ist für den

44 Vgl. dazu Marti (Fn. 2), Rz. 22 f. mit weiteren Hinweisen.
Zusammenhalt des Landes sehr wichtig.45

25 Rechtsgleichheitsfragen stellen sich im Föderalismus aber nicht nur im Bereich der Gesetzgebung, sondern auch im Bereich der Rechtsanwendung - und zwar auch bei der Anwendung von Bundesrecht. ${ }^{6}$ Grundsätzlich ist anerkannt, dass die Rechtsgleichheit im strengen Sinn beim Gesetzesvollzug nur gilt, wenn Behörden des gleichen Gemeinwesens entscheiden. Wenn Behörden verschiedener Gliedstaaten das Bundesrecht unterschiedlich anwenden, muss dies daher grundsätzlich als Konsequenz der dezentralisierten Rechtsanwendung hingenommen werden, welche in der Schweiz auch wichtiges Element der politischen Mitgestaltung der Kantone im Bund gilt. Allerdings ist es in allen Bundesstaaten Aufgabe der Bundesbehörden, im Rahmen ihrer Aufsicht und namentlich auch ihrer Rechtsprechung für eine korrekte und möglichst gleichmässige Rechtsanwendung $\mathrm{zu}$ sorgen. 47 Aber auch in diesem Punkt gibt es wesentliche Unterschiede zwischen den Staaten am Bodensee. Während das Bundesverfassungsgericht in Karlsruhe bei der Anwendung von Bundesrecht «eine im Wesentlichen einheitliche» Praxis der Länder verlangt, belässt das Schweizer Bundesgericht den Kantonen einen gewissen Anwendungsspielraum, soweit das Gesetz dies zulässt

45 Vgl. dazu Rainer J. Schweizer/Lucien Müller in St. Galler BV-Kommentar (3. Aufl. 2014), Art. 43a Rz. 16 ff. mit zahlreichen Hinweisen.

46 Vgl. dazu nun eingehend die Beiträge zum Thema «Rechtsanwendungsgleichheit in Mehrebenensystemen» von Wolfram Cremer und Benjamin Schindler in: Veröffentlichungen der Vereinigung der Deutschen Staatsrechtslehrer, Band 78, Berlin/New York 2018, S. 117 ff., 167 ff. (mit der anschliessenden Diskussion, S. $211 \mathrm{ff}$.).

47 Vgl. dazu Marti (Fn. 2), Rz. 24 f. mit weiteren Hinweisen. 
und dafür gute Gründe bestehen (z.B. unterschiedlicher Problemdruck, unterschiedliche politische, rechtliche oder kulturelle Rahmenbedingungen), womit z.B. - anders als in Deutschland - eine unterschiedliche Praxis der Kantone zur Anwendung des privilegierten Tatbestands des geringfügigen Eigengebrauchs im Cannabis-Strafrecht toleriert wurde. 48 Diese unterschiedliche Strenge bei der Frage der Rechtsanwendungsgleichheit im Bundesstaat ist natürlich selber ebenfalls auf unterschiedliche rechtskulturelle Hintergründe bzw. divergierende Föderalismuskonzepte zurückzuführen (eher unitarischer Föderalismus einerseits, ausgesprochener Wettbewerbs- und Vollzugsföderalismus andererseits).

\section{Rechtsstaatliche Defizite in kleinräumigen Verhältnissen}

Ein anderes Problem föderalistischer Systeme können die rechtsstaatlichen Defizite in kleinräumigen Strukturen bilden. So ist es namentlich in kleinen Gliedstaaten und Gemeinden nicht ohne Weiteres möglich, die für eine unabhängige Rechtsprechung nötigen Organe zur Verfügung zu stellen. In den Gemeinden bestehen denn auch in allen Staaten um den Bodensee regelmässig keine Rechtsprechungsorgane mit richterlicher Unabhängigkeit. Dies ist an sich auch nicht nötig, da unabhängige Gerichte auf der Gliedstaatsebene zur Verfügung stehen. Allerdings können sich daraus insofern Rechtsschutzdefizite ergeben, als gewisse Gemeindeentscheide - namentlich in der Schweiz durch die ausgeprägten Gemeindeautonomie - vor einer richterli-

48 Siehe das Urteil des Bundesverfassungsgerichts 90, 145 (169) und demgegenüber BGE 124 IV 184 E. 2b; dazu Schindler (Fn. 46), S. 177 ff.,198 ff. und Marti (Fn. 2), Rz. 25. chen Korrektur teilweise geschützt sind, was in der Schweiz etwa für die heiklen Fragen der kommunalen Bauordnungen (inkl. Ästhetikvorschriften) gilt. Eigentliche Gemeindegerichte einzuführen (nicht nur kommunale Schlichtungsstellen), wäre aber eine klare Überforderung der Rechtsstaatsstruktur. Solche gab es zwar in der mittelalterlichen Ordnung im ganzen deutschen Sprachraum, doch waren dies nicht unabhängige Gerichtsbehörden im heutigen Sinn, sondern meist allgemeine Verwaltungsbehörden mit umfassenden Kompetenzen. Heute muss es aus den erwähnten Gründen genügen, dass in lokalen Angelegenheiten eine Einsprache-Möglichkeit auf kommunaler Ebene gegeben ist (was allerdings nicht immer der Fall ist) und die Kognition der Verwaltungsgerichte auf Gliedstaatsebene nicht zu sehr eingeschränkt wird (weder rechtlich noch faktisch). 49

27 Aber nicht nur in den Gemeinden, auch in kleineren Gliedstaaten kann die Gewährleistung einer wirklich unabhängigen Justiz Probleme bieten. $\mathrm{Zu}$ denken ist etwa an den Kanton AppenzellInnerrhoden mit lediglich rund $16^{\circ}$ ooo Einwohnern. Dieser Halbkanton muss ebenfalls eine vollständige Behördenstruktur mit Parlament, Regierung und unabhängigen Gerichten (zwei Instanzen) zur Verfügung stellen. ${ }^{50}$ Ich erinnere mich an einen Besuch im Jahr 2001 beim damaligen Kantonsgerichtsschreiber Caius Savary (heutiger Bezirksgerichtspräsi-

$49 \overline{\text { Vgl. dazu Marti (Fn. 2), Rz. 27. Das Zürcher }}$ Verwaltungsgericht hat aus diesem Grund in den letzten Jahren seine Kognitionspraxis in diesem Bereich geändert; vgl. dazu Entscheid VB.2013.00468 vom 17. Dezember 2013, publiziert in $\mathrm{ZBl}$ 2014, S. 448 ff. (mit Anmerkungen des Autors).

50 Vgl. dazu auch Marti (Fn. 2), Rz. 26. 
dent Appenzell) im damals neuen Justizgebäude in Appenzell. Er zeigte mir die verschiedenen Abteilungen: die Kantonspolizei im Erdgeschoss, die Kriminalpolizei im zweiten Stock, die Staatsanwaltschaft im dritten Stock und die beiden Gerichte im vierten Stock und erläuterte mir, dass je gegenseitige Stellvertretungsregelungen bestünden, was immer wieder zu Ausstandsproblemen führe. Auch die Ernennung der Richterinnen und Richter für die traditionell sehr grossen Kammern (am Kantonsgericht Siebnerkammer als Regel) ist nicht einfach, weil das aktive und passive Wahlrecht im kleinen Kanton nötig ist; es funktioniert wohl nur deshalb, weil grossmehrheitlich immer noch Laienrichter tätig sind.51 In diesem Zusammenhang interessant: Der daher besonders wichtige Gerichtsschreiber musste bis vor nicht allzu langer Zeit noch vom Parlament gewählt werden! 52

28 Ähnliche Probleme bestehen natürlich auch im 38‘0oo Einwohner starken Fürstentum Liechtenstein, wobei dort nicht nur eine Gliedstaatenorganisation, sondern eine Gesamtstaatsorganisation geschaffen und unterhalten werden muss (mit allen erforderlichen Ministerien, auch einem Aussenministerium und fünf Gerichtsinstanzen). In Liechtenstein aber wusste man sich zu helfen. Quasi als Kontrapunkt zum Schweizer Abwehrre-

$51 \overline{\text { Vgl. zur heutigen Organisation des } 13 \text { Mitglieder }}$ umfassenden Kantonsgerichts als zweiter Instanz im Zivil- und Strafrecht und als Verwaltungsgericht Art. 10 und 11 des Gerichtsorganisationsgesetzes vom 25. April 2010 (GS 173.000).

52 Vgl. den früheren, erst 1994 geänderten Wortlaut von Art. 28 Abs. 2 der Verfassung des Kantons Appenzell Innerrhoden vom 24. November 1872 und dazu die Gewährleistungsbotschaft des Bundesrates vom 2. November 1994, BBl 1995 I 969 ff., insbesondere S. 974. flex gegen fremde Richter besteht in Vaduz eine alte Tradition fremder Richter. Schon bei der Staatsgründung bildete das fürstliche Hofgericht in Wien Appellationsinstanz, später aus Mangel an einheimischen Juristen das Oberlandesgericht Innsbruck. Mit der Verfassung von 1921 wurden die Gerichte «ins Ländle heimgeholt», aber ausdrücklich auch die Wahl ausländischer Richter zugelassen. Lediglich für die obersten Gerichte (Verwaltungs- und Staatsgerichtshof) besteht die Einschränkung, dass die Mehrheit der Richter und der Präsident des Staatsgerichtshofs liechtensteinische Staatsangehörige sein müssen. Mit diesem besonderen System hat Liechtenstein gute Erfahrungen gemacht: Es konnte sehr gute und wirklich unabhängige Richterpersönlichkeiten aus der Schweiz und Österreich gewinnen, die das Fürstentum immerhin selber aussuchen konnte. $53 \mathrm{Zu}$ reden gab in letzter Zeit lediglich der grosse Einfluss von Fürst und Regierung im Richterwahl-Vorbereitungsgremium (Art. 96 der Verfassung gemäss der seinerzeit sehr umstrittenen Verfassungsreform von 2003). Diese Reform löste denn auch ein Verfahren im Europarat aus, welches inzwischen aber abgeschlossen ist. 54

53 Vgl. dazu «Keine Angst vor fremden Richter», NZZ vom 18. Juli 2019, S. 13 und überdies die Untersuchung von Anna-Carolina Perrez, Fremde Richter. Die Rechtsprechung im Fürstentum Liechtenstein unter dem Einfluss schweizerischer und deutsch-österreichischer Richter 1938-1945, Zürich 2015.

54 Vgl. dazu «Europarat-Monitoring für Liechtenstein?», sda-Meldung in NZZ vom 12. September 2003. Der Europarat verzichtete 2007 schliesslich auf ein solches Monitoring zugunsten periodischer Länderberichte. Der neue Art. 96 der Liechtensteiner Verfassung sieht für Richterwahlen ein empfehlendes Auswahlgremium mit Vetorecht des Fürsten vor. Die entsprechende neue Regelung erfolgte aufgrund des Urteil des EGMR 28396/95 vom 28. Oktober 1999 (Wille gegen 

Richterinnen und Richtern könnte jedenfalls auch in kleineren Schweizer Kantonen die Auswahlmöglichkeiten und die Unabhängigkeit der Justizbehörden verbessern und hätte wohl mehr Realisierungschancen als die mit der Justizreform möglich gewordene Bildung von gemeinsamen interkantonalen Gerichten (Art. 191b BV). Die in der Schweiz traditionell politischen Richterwahlen (durch das Parlament oder gar durch das Volk), welche die besondere Legitimation der Richterinnen und Richter und auch eine breite Repräsentation in den Gerichten sichern sollen, werden heute im Übrigen in unserem Land auch nicht mehr als gottgegeben angesehen: Im Bund und in vielen Kantonen sind Fachgremien eingeführt worden, welche die Richterwahlen vorbereiten, und aufgrund einer Volksinitiative wird sogar die Wahl der Richter durch das Los diskutiert. 55

\section{Fazit und Schlussbemerkungen}

30 Die gemachten Darlegungen zeigen, dass Föderalismus und Rechtsstaatlichkeit in den Staaten um den Bodensee eine - allerdings unterschiedlich lange und verschieden ausgeprägte - Tradition besitzen und sich daraus hin und wieder auch Konflikte oder zumindest Spannungsverhältnisse ergeben haben. Herausforderungen können sich namentlich auch aus neueren Entwicklungen in Politik, Wirt-

Liechtenstein), in welchem der Gerichtshof eine Konventionsverletzung durch das Fürstentum feststellte, weil der Fürst die Ernennung eines Richters ablehnte, welcher in einem Vortrag eine dem Fürsten nicht genehme Auffassung über die Auslegungskompetenz des Staatsgerichtshofs vertrat.

55 Vgl. dazu auch die Hinweise bei Marti (Fn. 2), Rz. 28 und 30 sowie den Text der sog. "JustizInitiative» in BBl 2018, S. 2669 ff. schaft und Gesellschaft ergeben. Dem Rechtsstaat und seiner Weiterentwicklung ist weiterhin aufmerksam Sorge zu tragen. Den bei uns heute zum Glück seltenen Anfechtungen des Rechtsstaats ist konsequent und überzeugend entgegenzutreten und es sollten Information und Bildung in diesem Bereich jedenfalls vermehrt gefördert werden. ${ }^{6} 6$ Hinsichtlich des Föderalismus müssen eine gewisse Uneinheitlichkeit und allenfalls auch Entwicklungsverzögerungen zugunsten einer besseren Akzeptanz und der Möglichkeit kreativer Neuerungen auch in der Gesetzgebung in Kauf genommen werden (die Gliedstaaten als «gesetzgeberische Versuchslabors»). Verbesserungen im föderalistischen System sind aber immer wieder zu prüfen: So sollte allenfalls in Deutschland und Österreich etwas mehr Wettbewerb zwischen den Ländern ermöglicht werden und in der Schweiz vielleicht eher etwas weniger.57 Insgesamt aber kann sicher gesagt werden, dass die Länder am Bodensee von den meist kleinräumigen, überschaubaren und rechtlich geordneten Verhältnissen sehr profitiert haben und diese sicher nicht aufgeben wollen. Die rechtsstaatlich-föderalistische Ordnung ermöglicht zusammen mit den unterschiedlichen Formen der demokratischen Partizipation eine auf ein gutes

$56 \overline{\text { Vgl. dazu das auch auf andere Rechtsstaaten }}$ übertragbare Plädoyer von Markus Müller, Perspektiven der schweizerischen Rechtsstaatlichkeit, in dem in Fn. 2 erwähnten Sammelwerk, Band II, S. $1147 \mathrm{ff}$.

57 Vgl. dazu eingehend Peter Bussjäger/Stefan August Lütgenau, Föderalismus im 21. Jahrhundert. Effizienz und Verantwortung im modernen föderalistischen Staat, Innsbruck 2012; zu den sich wandelnden Bedingungen und Herausforderungen des schweizerischen Föderalismus insbesondere auch Bernhard Waldmann, Perspektiven des schweizerischen Föderalismus, in dem in Fn. 2 erwähnten Sammelwerk, Band I, S. 797 ff. 
Zusammenleben ausgerichtete menschengerechte Organisation, welche insbesondere auch sehr gut und erfolgreich der Verwirklichung des Gemeinwohls dient. Sie hebt sich dadurch auch äusserst positiv von anderen Staatsformen ab, welche auf zentralistische und autoritäre Machtausübung setzen. Diese haben heute zwar in verschiedenen Weltgegenden wieder Konjunktur und mögen kurzfristig auch einstweilige wirtschaftliche Erfolge haben - aber kaum auf Dauer, wie gerade Ereignisse in der letzten Zeit zeigen. Auch wenn die Zukunft immer unsicher ist und globale Veränderungen (z.B. Klimawandel, Migration) uns auch am schwäbischen Meer erreichen, dürfen wir daher die Hoffnung behalten, in der schönen Umgebung des Bodensees auch auf längere Sicht in einer der vergleichsweise besten Welten zu leben. 